\title{
Inflammatory Cell Infiltrates in Acute and Chronic Thoracic Aortic Dissection
}

\author{
Darrell Wu, MD ${ }^{1,2,3, \dagger}$, Justin C. Choi, $\mathrm{MD}^{1,2, \dagger}$, Aryan Sameri, $\mathrm{BS}^{1,2}$, Charles G. Minard, PhD ${ }^{4}$, \\ Joseph S. Coselli, $M D^{1,2}$, Ying H. Shen, $M D, P h D^{1,2}$, Scott A. LeMaire, $M D^{1,2,3 *}$ \\ ${ }^{1}$ Division of Cardiothoracic Surgery, Michael E. DeBakey Department of Surgery, Baylor College of Medicine, Houston, Texas; \\ ${ }^{2}$ Department of Cardiovascular Surgery, The Texas Heart Institute, Houston, Texas; ${ }^{3}$ Department of Molecular Physiology and \\ Biophysics, Baylor College of Medicine, Houston, Texas; and ${ }^{4}$ Dan L. Duncan Institute for Clinical and Translational Research, Baylor \\ College of Medicine, Houston, Texas
}

\begin{abstract}
Background: Thoracic aortic dissection (TAD) is a highly lethal cardiovascular disease. Injury to the intima and media allows pulsatile blood to enter the media, leading to dissection formation. Inflammatory cells then infiltrate the site of aortic injury to clear dead cells and damaged tissue. This excessive inflammation may play a role in aneurysm formation after dissection. Methods: Using immunohistochemistry, we compared aortic tissues from patients with acute TAD $(n=11)$, patients with chronic TAD $(n=35)$, and donor controls $(n=20)$ for the presence of CD68+ macrophages, neutrophils, mast cells, and CD3 + T lymphocytes. Results: Tissue samples from patients with acute or chronic TAD generally had significantly more inflammatory cells in both the medial and adventitial layers than did the control samples. In tissues from patients with acute TAD, the adventitia had more of the inflammatory cells studied than did the media. The pattern of increase in inflammatory cells was similar in chronic and acute TAD tissues, except for macrophages, which were seen more frequently in the adventitial layer of acute TAD tissue than in the adventitia of chronic TAD tissue. Conclusions: The inflammatory cell content of both acute and chronic TAD tissue was significantly different from that of control tissue. However, the inflammatory cell profile of aneurysmal chronic TAD was similar to that of acute TAD. This may reflect a sustained injury response that contributes to medial degeneration and aneurysm formation.

Copyright @ 2013 Science International Corp.
\end{abstract}

\section{Key Words}

Inflammation - Thoracic aortic dissection

Macrophage · Mast cell · Neutrophil · T lymphocyte

\section{Introduction}

Aneurysm formation after thoracic aortic dissection (TAD) is a deadly cardiovascular disease and a major cause of morbidity and mortality [1]. Aortic dissections occur when pulsatile blood enters an intimal tear and causes the medial layer to split along the length of the aorta. Weakening of the aortic wall can be caused by medial degeneration, which is characterized by vascular smooth muscle cell depletion and elastic fiber depletion and fragmentation $[2,3]$. Concurrently, inflammatory cells can infiltrate the injured aortic wall, clear the dead cells, remove damaged matrix proteins, and remodel the extracellular matrix [4]. However, uncontrolled inflammatory processes can lead to tissue destruction in the aorta $[5,6]$, which in turn may lead to the formation of an aneurysm after aortic dissection. The role of inflammation after dissection as a cause of aneurysm formation has not been well characterized.

Previous studies have shown that CD68+ macro-

*Corresponding author:

Scott A. LeMaire, MD

Division of Cardiothoracic Surgery

Michael E. DeBakey Department of Surgery

Baylor College of Medicine

One Baylor Plaza, BCM 390, Houston, TX 77030

Tel.: +1 832355 9942, Fax: +1 832355 9928, E-Mail: slemaire@bcm.edu

${ }^{\dagger}$ These authors contributed equally to this study. 
Table 1. Primary Antibodies Used for Immunohistochemical Analysis

\begin{tabular}{llllll}
\hline Antibody & Cell type & Source & Clone & Manufacturer & Dilution factor \\
\hline CD 68+ & Macrophage & Monoclonal mouse anti-human & KP1 & Dako (Carpinteria, CA) & $1: 50$ \\
Mast cell tryptase & Mast cell & Monoclonal mouse anti-human & AA1 & Dako & $1: 200$ \\
Neutrophil elastase & Neutrophil & Monoclonal mouse anti-human & NP57 & Dako & $1: 125$ \\
CD3 + & T Lymphocyte & Monoclonal mouse anti-human & F7.2.38 & Dako & $1: 50$ \\
\hline
\end{tabular}

phages [7], neutrophils [8], mast cells, and CD3+ [5,6] and CD4+ T lymphocytes [9] are significantly increased in the aortic wall of patients with abdominal aortic aneurysms or ascending thoracic aortic aneurysms (TAAs) (both heritable and sporadic), as well as in patients with Type A dissections $[5,6]$. However, the inflammatory infiltrates present in acute TAD and descending TAA due to chronic TAD are not well documented. In this study, we examined aortic tissues from patients with acute ascending TAD or descending aneurysms after TAD for the presence of CD68+ macrophages, neutrophils, mast cells, and CD3 + T lymphocytes in both early and late phases of the dissection. We hypothesized that chronic TAD tissues would exhibit reduced inflammation and an altered inflammatory cell profile compared to acute TAD tissues.

\section{Materials and Methods}

\section{Study Enrollment and Tissue Collection}

The institutional review board at Baylor College of Medicine approved this study. Informed written consent was obtained from all subjects. We enrolled 46 patients who underwent repair of an acute or chronic TAD and who did not have aortitis, dissection variants such as intramural hematoma or penetrating aortic ulcer, or a dissection caused by trauma. Tissue samples obtained within 14 days of TAD onset were considered acute $(n=11)$, whereas those obtained more than 60 days after TAD onset were considered chronic $(n=35)$; we did not enroll patients in whom tissue samples would be obtained during the subacute phase (ie, between 14 and 60 days after TAD onset). During dissection repair, we excised tissue samples from the outer wall of the false lumen. Control aortic tissues $(n=20)$ were obtained from organ or tissue donors who had no aortic aneurysm, dissection, coarctation, or prior aortic repair and no evidence of sepsis.

\section{Histology and Immunohistochemical Staining}

Aortic tissues were paraffin-embedded and sectioned. Endogenous peroxidase activity in aortic sections was quenched by $3 \%$ hydrogen peroxide treatment. Citric acid antigen retrieval was performed. Tissue sections were blocked in 5\% normal horse serum and incubated overnight with primary antibodies (Table 1). Samples were then incubated with the appropriate biotin-conjugated anti-mouse IgG secondary antibodies (Vector Laboratories, Inc., Burlingame, CA, USA). Normal mouse immunoglobulin $G$ (Vector Laboratories) served as the negative control for immunostaining. Inflammatory cells were visualized by using peroxidase substrate 3,3'-diaminobenzidine (DAB; Vector Laboratories), and cell nuclei were counterstained with hematoxylin (Sigma Aldrich, St. Louis, MO, USA). Image Pro-Plus 4.5 (Leica Microsystems, Bannockburn, IL, USA) was used to quantify the positive-staining inflammatory cells within the medial and adventitial layers. Three microscopic fields $(400 \times)$ were randomly selected from each layer for analysis. Positive-staining areas were then normalized to an observed tissue area within the same sample.

\section{Statistical Analysis}

All quantitative data are presented as the mean \pm standard deviation. Data were analyzed with SPSS software, version 20.0 (SPSS Inc., Chicago, IL, USA). The difference between the mean ratios of positively stained area $\left(\mu \mathrm{m}^{2}\right)$ to observed tissue area $\left(\mu \mathrm{m}^{2}\right)$ among the groups was compared by using the MannWhitney or Kruskal-Wallis nonparametric test with Bonferroni correction. The representative non-normal distribution of the positive-staining area was depicted by using boxplots with a five-point summary scale.

\section{Results and Discussion}

In response to aortic injury, inflammatory cells infiltrate the aortic wall to aid in tissue repair [4]. In this study, we characterized the inflammatory infiltrate observed in aortic tissues from acute and chronic TAD patients by means of immunohistochemistry; all TAD tissues showed significantly more CD68+ macrophages, neutrophils, mast cells, and CD3 + T lymphocytes in both the medial and adventitial layers as compared to the same vessel layer in aortic tissues from controls (Figs. $1 \mathrm{~A}$ and $1 \mathrm{~B}, 2 \mathrm{~A}$ and $2 \mathrm{~B}, 3 \mathrm{~A}$ and $3 \mathrm{~B}$, and $4 A$ and $4 B$, respectively). Moreover, we found a greater abundance of all inflammatory cell types in the adventitia than in the media (Figs. 1C, 2C, 3C, and 4C, respectively); this finding suggests that inflammatory cells infiltrate the aortic wall from the vasa vasorum into the media [10]. This increased inflammatory infil- 


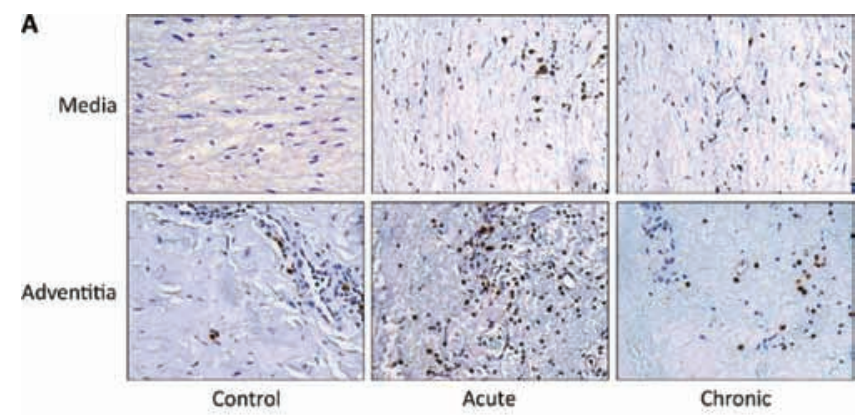

B
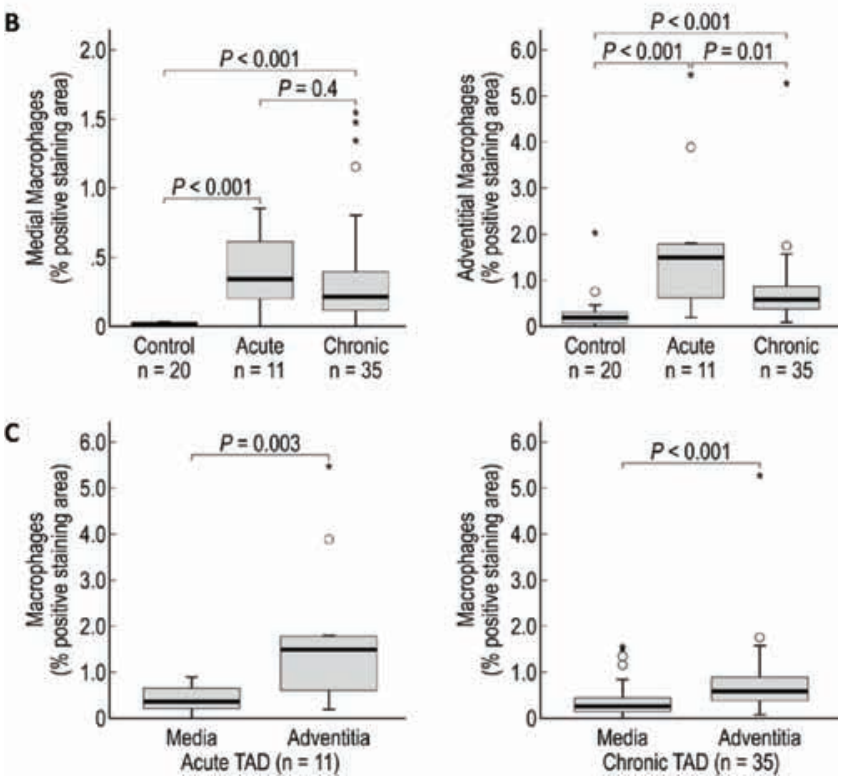

Figure 1. $\mathrm{CD} 68+$ macrophages are increased in the media and adventitia of acute and chronic TAD tissues. A. Immunohistochemistry staining and comparison of macrophages in the medial and adventitial layers of the aortas from tissue samples from donor controls and acute and chronic TAD patients. Original magnification, $200 \times$. B. Comparison of the positivestaining areas of control, acute, and chronic dissection tissues in the media and adventitia. C. Comparison of the positivestaining areas of the media and adventitia in acute and chronic TAD. The tips of the projecting bars represent the minimum and maximum values, and the box depicts the interquartile range, with the solid middle line representing the median. Circles and asterisks represent $1.5 \times$ and $3 \times$ the interquartile range, respectively.

trate at the site of either acute or chronic dissection suggests that an uncontrolled or chronic inflammatory response may contribute to aortic destruction and maladaptive remodeling of the aortic wall. Our findings support previous reports of a similar increase in inflammatory infiltrates in thoracic and abdominal aortic aneurysms $[5,6]$, suggesting a possible shared mechanism of aortic degeneration among thoracic and abdominal aortic aneurysms and acute and chronic TAD.

\section{Patient Characteristics}

The clinical characteristics and demographics of the TAD patients and control donors are shown in Table 2. Patients with acute TAD tended to be younger, and the percentage of smokers was similar across the three groups. No patient in the chronic TAD group had diabetes. As expected, the time to surgery was longer for the chronic TAD patients than for the acute TAD patients $(5 \pm 3$ days versus $1730 \pm 2088$ days), and we had a higher number of ascending aorta samples $(n=10)$ collected from acute TAD patients and a higher number of descending aorta samples $(n=20)$ from chronic TAD patients. The aortic diameters were similar for acute and chronic TAD patients.

\section{Macrophages in TAD Tissues}

Macrophages are one of the most abundant inflammatory cells in the media and adventitia of abdominal aortic aneurysms (AAAs), TAA $[5,6]$, and TAD tissues [5]. Because they secrete proteases such as collagenases, elastase, and matrix metallopeptidase-9 (MMP-9) that directly destroy the extracellular matrix [7] and cytokines and chemokines such as interleukin 6 (IL-6) and monocyte chemotactic protein-1 (MCP-1) that recruit cells, macrophages are instrumental in maintaining and amplifying the inflammatory cascade [11]. Using a marker for phagocytic cells, our immunohistochemical analysis showed that more areas in the media and adventitia in both acute and chronic TAD tissues stained positively for CD68+ macrophages than did areas of control tissues (Fig. 1A). Although CD68 is not a macrophage-specific antigen, in this instance, sampling of the outer wall minimizes the presence of any lipid-rich regions, limiting any cross-reactivity with CD68+ smooth muscle cells [12]. On quantification, we also found a significant increase in CD68+ macrophage content in the media and adventitia of acute and chronic TAD tissues (Fig. 1B) as compared to that in control tissues. Medial macrophage content did not differ significantly between acute and chronic TAD tissues, but we found significantly higher levels of macrophages in the adventitia of acute TAD tissues than in chronic TAD tissues. In both acute and chronic TAD tissues, the adventitia contained significantly more areas that stained positive for macrophages than did the media. These find- 
Table 2. Characteristics of TAD Patients and Control Tissue Donors

\begin{tabular}{|c|c|c|c|c|}
\hline & Control $n=20$ & Acute TAD $n=11$ & Chronic TAD $n=35$ & $p$-value ${ }^{* *}$ \\
\hline Age (years)* $^{*}$ & $57 \pm 9$ & $49 \pm 16$ & $54 \pm 14$ & 0.2 \\
\hline Hypertension & $11(55 \%)$ & $9(82 \%)$ & 32 (91\%) & 0.006 \\
\hline Smoking & $8(40 \%)$ & $5(46 \%)$ & $19(54 \%)$ & 0.60 \\
\hline Diabetes & $6(30 \%)$ & $2(18 \%)$ & 0 & 0.001 \\
\hline Stroke & $8(40 \%)$ & 0 & $2(6 \%)$ & 0.002 \\
\hline Coronary artery disease & 0 & $1(9 \%)$ & $9(26 \%)$ & 0.02 \\
\hline Peripheral vascular disease & 0 & 0 & $4(11 \%)$ & 0.3 \\
\hline Chronic obstructive pulmonary disease & $1(5 \%)$ & 0 & $4(11 \%)$ & 0.6 \\
\hline Confirmed diagnosis of Marfan syndrome & 0 & $2(18 \%)$ & $5(14 \%)$ & 0.1 \\
\hline Bicuspid valve disease & 0 & 0 & 0 & 1.00 \\
\hline Aortic diameter $(\mathrm{cm})^{*}$ & & $5.5 \pm 1.6$ & $6.0 \pm 1.4$ & 0.3 \\
\hline Interval to surgery from time of dissection (days)* & & $5 \pm 3$ & $1730 \pm 2088$ & $<0.001$ \\
\hline \multicolumn{5}{|l|}{ Sample site } \\
\hline Ascending aorta & $10(50 \%)$ & $10(91 \%)$ & $15(43 \%)$ & \\
\hline Descending aorta & $10(50 \%)$ & $1(9 \%)$ & $20(57 \%)$ & 0.02 \\
\hline
\end{tabular}

*Data are presented as mean \pm standard deviation.

** $p$-values comparing groups by using Kruskal-Wallis tests (continuous variables) or Fisher's exact test (discrete variables).

ings support the role of macrophages in ongoing aortic tissue destruction after dissection formation.

When evaluating the potential effects of macrophages, it is important to consider the two different subpopulations of macrophages: the proinflammatory M1 macrophages and the anti-inflammatory M2 macrophages. Studies have shown that an extensive presence of the cytotoxic M1 subtype can further contribute to tissue injury and destruction because these macrophages can release reactive oxygen species and nitric oxide synthase [13]. In contrast, M2 macrophages have been shown to resolve inflammation by inhibiting $T$ cell proliferation, phagocytizing apoptotic neutrophils, reducing the production of proinflammatory cytokines, and secreting and stabilizing matrix components [13]. Therefore, comparing the levels of proinflammatory M1 macrophages and anti-inflammatory M2 macrophages in TAD tissue could help determine whether or not a chronic inflammatory state is likely to lead to an altered tissue homeostasis dominated by destructive factors.

\section{Neutrophils in TAD Tissues}

Neutrophils are key regulators of sterile vascular inflammation [14] and are capable of secreting serine proteases, cathepsins, and reactive oxygen intermediates that can damage the extracellular matrix $[8,14]$. In a mouse study, neutrophil depletion prevented AAA development [8], suggesting that neutrophil recruit- ment is critical for the development of aortic aneurysms. Furthermore, doxycycline therapy has been shown to improve proteolytic balance by reducing the neutrophil content in patients undergoing elective repair of AAA [15]. In the present study, we observed an increase in neutrophil cells in the media and adventitia of both acute and chronic TAD tissues (Fig. 2B). Our findings support those of Cohen et al. [16], who also reported an increase in neutrophil levels in AAA. Thus, we believe that neutrophils may play a role in the inflammatory cascade after an acute dissection and that increased neutrophil levels in chronic TAD tissues could suggest ongoing vascular injury, reflecting an acute-on-chronic inflammatory response that contributes to aneurysm formation.

\section{Mast Cells in TAD Tissues}

Like macrophages and neutrophils, mast cells have been shown to play a significant role in the development of AAA. Mast cells are capable of secreting chymases, which can activate matrix metalloproteinases, and angiotensin II, both of which contribute to aneurysm formation $[17,18]$. Additionally, mast cells can secrete tryptases and proinflammatory signaling factors, such as interferon-gamma (IFN $\gamma$ ), IL- 6 , and tumor necrosis factor- $\alpha$ (TNF $\alpha$ ), which can lead to an increase in monocyte infiltration, chemokine production, and vascular cell injury [19]. Furthermore, treatment with tranilast, a mast cell degranulation inhibitor, attenu- 

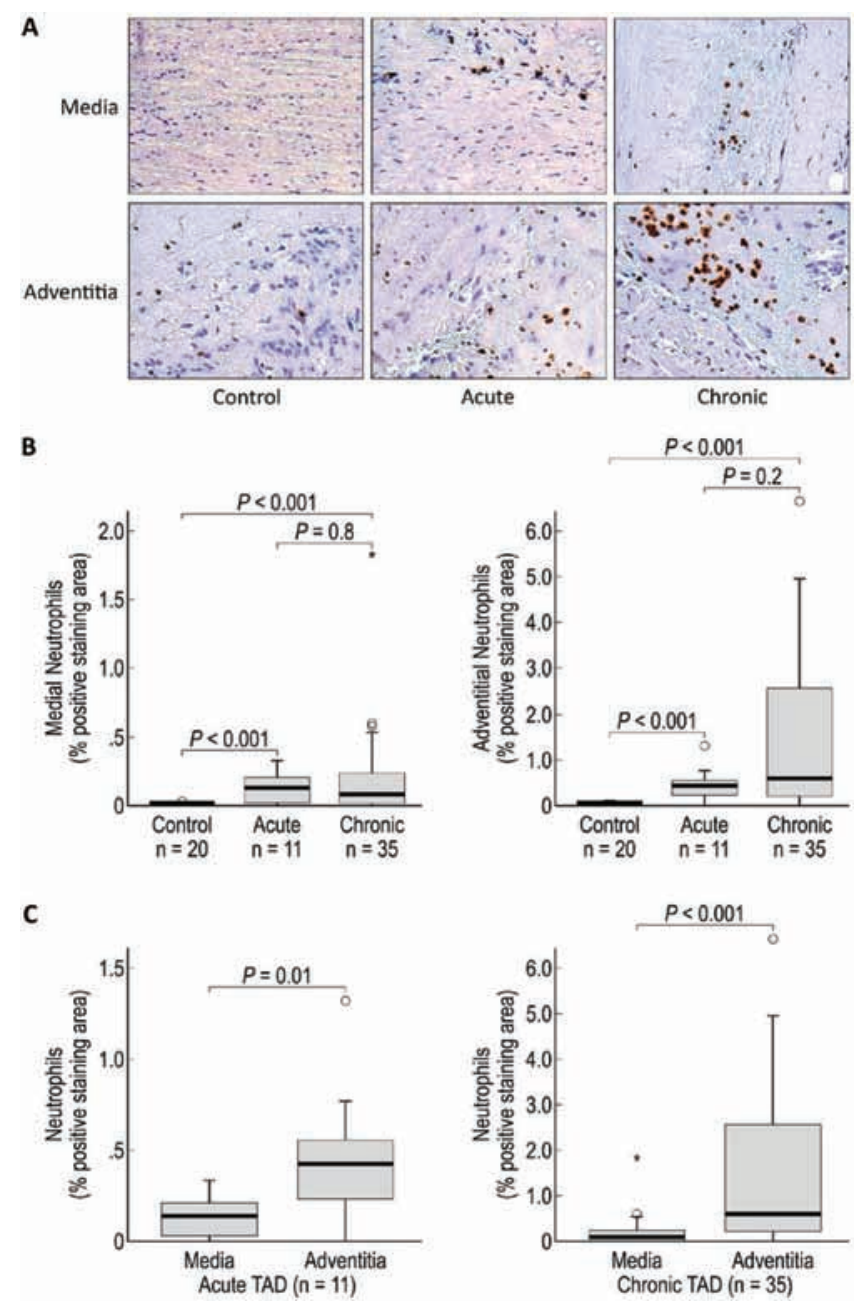

Figure 2. Neutrophils are increased in the media and adventitia of acute and chronic TAD tissues. A. Immunohistochemistry staining and comparison of neutrophils in the medial and adventitial layers of the aortas from donor controls and acute and chronic TAD patients. Original magnification, 200×. B. Comparison of the positive-staining areas of control, acute, and chronic dissection tissues in the media and adventitia. C. Comparison of the positive-staining areas of the media and adventitia in acute and chronic TAD. The tips of the projecting bars represent the minimum and maximum values, and the box depicts the interquartile range, with the solid middle line representing the median. Circles and asterisks represent $1.5 \times$ and $3 \times$ the interquartile range, respectively.

ates aneurysm formation [20]. In the present study, we found a significant increase in mast cells in the aortic media and adventitia of both acute and chronic TAD tissues (Fig. 3B). Given the critical role of mast cells in vascular destruction, we believe they may be important contributors to the continued dilation and destruction of the aortic wall.
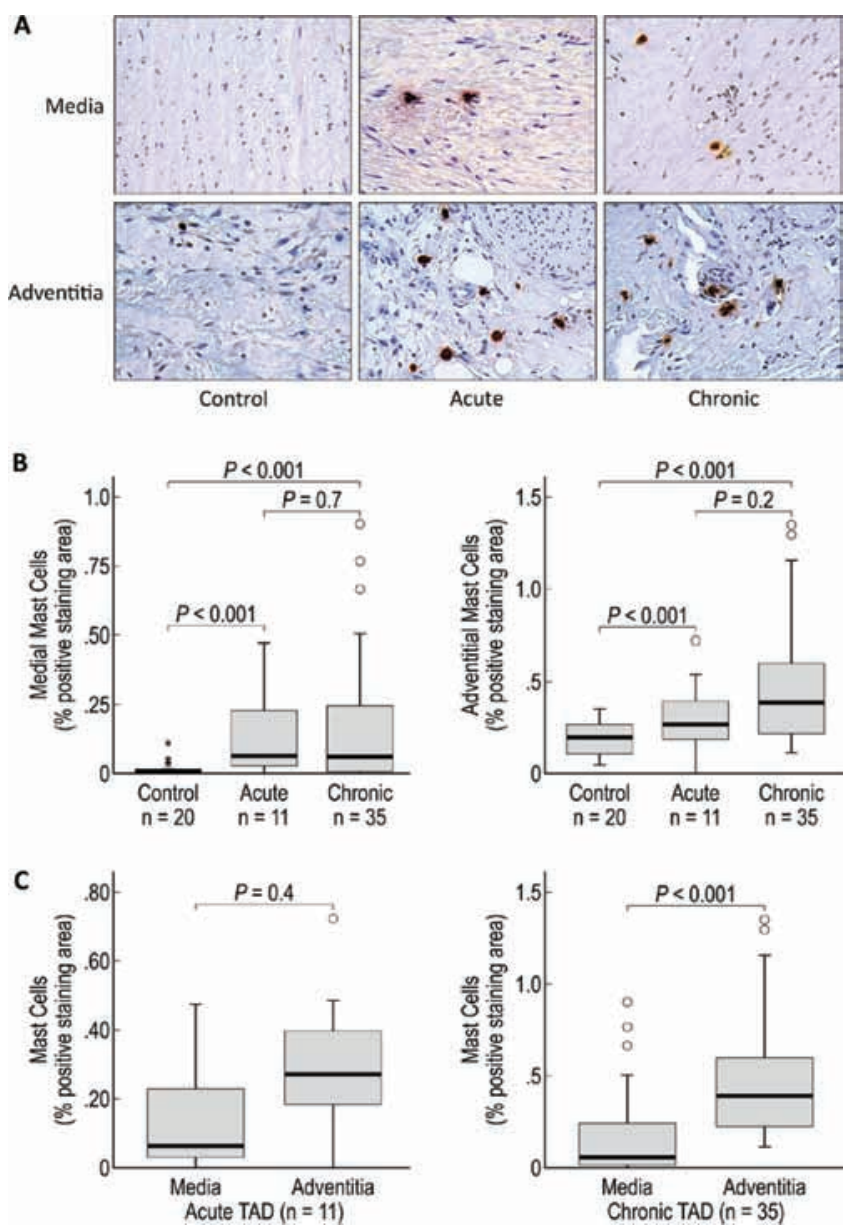

Figure 3. Mast cells are increased in the media and adventitia of acute and chronic TAD tissues. A. Immunohistochemistry staining and comparison of mast cells in the medial and adventitial layers of the aortas from donor controls and acute and chronic TAD patients. Original magnification, 200×. B. Comparison of the positive-staining areas of control, acute, and chronic dissection tissues in the media and adventitia. C. Comparison of the positive-staining areas of the media and adventitia in acute and chronic TAD. The tips of the projecting bars represent the minimum and maximum values, and the box depicts the interquartile range, with the solid middle line representing the median. Circles and asterisks represent $1.5 \times$ and $3 \times$ the interquartile range, respectively.

\section{CD3 $+T$ Lymphocytes in TAD}

CD3 + T lymphocytes are capable of secreting cytotoxic mediators such as Fas/FasL and perforin, which can cause cell death and have been reported to be the most prominent inflammatory cell in the media of TAD [6]. Furthermore, CD3 + T lymphocyte activation can lead to the secretion of proteases that can weaken the aortic wall. For example, contact between mast cells 


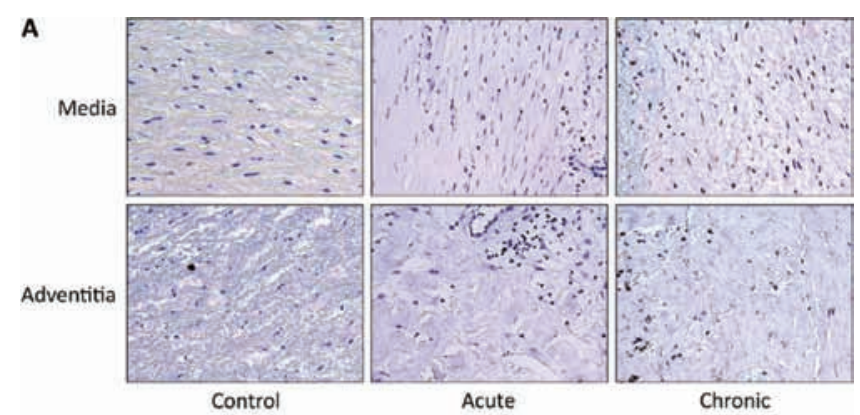

B
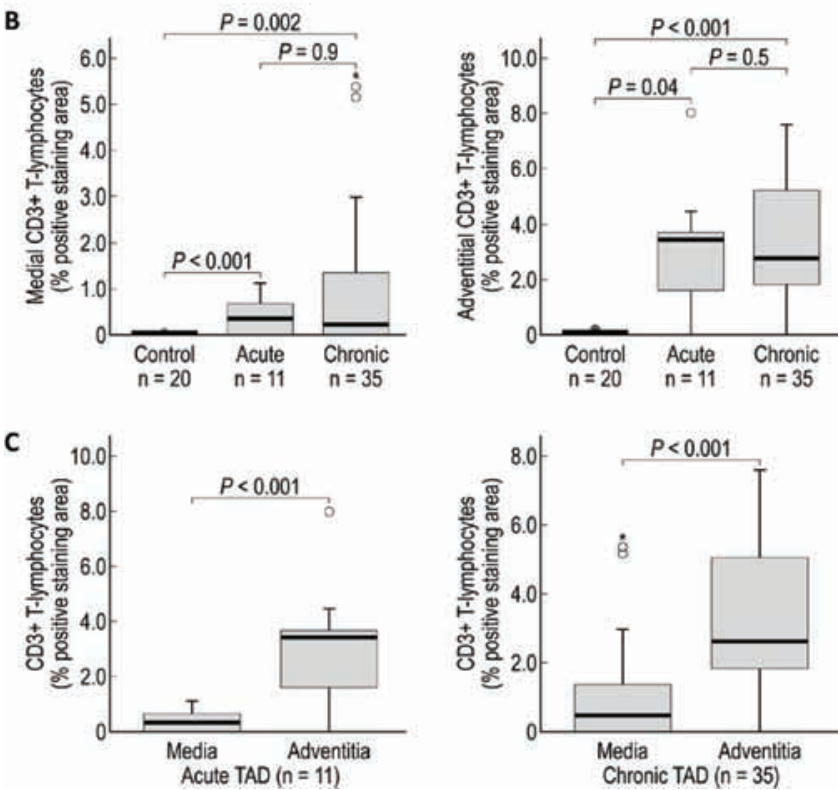

Figure 4. $\mathrm{CD} 3+\mathrm{T}$ lymphocytes are increased in the media and adventitia of acute and chronic TAD tissues. A. Immunohistochemistry staining and comparison of CD3 + T lymphocytes in the medial and adventitial layers of the aorta from donor controls and acute and chronic TAD patients. Original magnification, 200×. B. Comparison of the positive-staining areas of control, acute, and chronic dissection tissues in the media and adventitia. C. Comparison of the positive-staining areas of the media and adventitia in acute and chronic TAD. The tips of the projecting bars represent the minimum and maximum values, and the box depicts the interquartile range, with the solid middle line representing the median. Circles and asterisks represent $1.5 \times$ and $3 \times$ the interquartile range, respectively.

and T lymphocytes can result in the release of MMP-9 from mast cells [21]. In the current study, we found that CD3 + T lymphocytes were significantly increased in the media and adventitia of acute and chronic TAD tissues compared to control tissues (Fig. 4B). Our findings, combined with those showing increased CD3 $+\mathrm{T}$ lymphocyte levels in both sporadic and heritable ascending TAA and Type A dissections [6], suggest that the pathogenesis of sporadic and heritable aneurysms and dissection may share a common immune mechanism.

\section{Media versus Adventitia}

In the traditional view of vascular inflammation, chemoattraction results in the movement of immune cells through the endothelium to the media. However, growing evidence indicates that the adventitia may play a more prominent role in maintaining an inflammatory response [22]. The adventitia is a major site of inflammatory cell accumulation, and an extensive infiltration of macrophages has been linked to aortic aneurysm development [23]. In this study, we found significantly more CD68+ macrophages, neutrophils, and CD3 + T lymphocytes in the adventitia than in the media in both acute and chronic cases of TAD (Figs. 1C, 2C, and 4C). The abundance of inflammatory cells in the adventitia indicates that the adventitia is a dynamic microenvironment intimately involved in aortic wall homeostasis.

\section{Eosinophils}

One cell type that was not studied was eosinophils. Eosinophils are capable of degranulating cytotoxic proteins that can damage tissue, produce superoxide and transforming growth factor- $\beta$, and be stimulated by neutrophils to produce proinflammatory cytokines to further perpetuate an inflammatory response [24]. Despite these numerous functions, the precise role of eosinophils in causing aortic aneurysms or dissection is not well understood, although eosinophils are present in the media of acute ascending dissection, suggesting a potential role of eosinophils in causing aortic dissection [25].

\section{Study Limitations}

Tissue samples in the TAD group were obtained from patients who had other underlying diseases in addition to the dissection, and some patients experienced dissection after aneurysm formation. Thus, patient heterogeneity and comorbidity factors may have affected the inflammatory response to dissection; however, this study was not powered to assess the clinical correlations between the degree of inflammatory infiltration and patient comorbidities. Furthermore, we evaluated only end-stage aortic tissue; the role of inflammatory cells in the early stages of the disease process needs to be studied to determine whether their presence is a contributing factor to 
the initial development of TAD or solely a response to aortic injury after TAD. We also did not include an analysis of tissue from the interim subacute period after acute $(<14$ days) and before chronic ( $>60$ days) dissection. Although these definitions of acute and chronic are arbitrary, tissue is more friable and difficult to operate on during this time frame, suggesting that there may be an immense amount of remodeling. For this reason, one might expect there to be even larger amounts of inflammatory cells present in these subacute cases than in acute or chronic cases. Additionally, we did not delineate subpopulations of inflammatory cells. Finally, technical limitations of our analysis based on the mean ratios of positively stained area $\left(\mu \mathrm{m}^{2}\right)$ to observed tissue area $\left(\mu \mathrm{m}^{2}\right)$ precluded a comparison of the relative distribution between cell types. Despite these limitations, the results of our study support the important role of the inflammatory response in TAD.

\section{Conclusion}

We observed a significant increase in CD68+ macrophages, neutrophils, mast cells, and CD3 + T lymphocytes in the media and adventitia of acute and chronic TAD tissues. The pattern of increase in inflammatory cells was similar in acute and chronic dissection tissue. The significant difference between the number of inflammatory cells seen in the medial and adventitial layers suggests that the cells infiltrate the media through the vasa vasorum. Overall, this study suggests that inflammation may play a role in tissue destruction and the development of aortic aneurysm after dissection.

\section{Acknowledgments}

This study was supported by NIH Grants K08 HL080085 and R01 HL085341 (to S.A.L.). The Thoracic Aortic Disease Tissue Bank at Baylor College of Medicine was supported in part through the Tissue Banking Core of the Specialized Center of Clinically Oriented Research in Thoracic Aortic Aneurysms and Dissections (NIH P50 HL083794). Darrell Wu was supported by a training grant (NIH T32 HL007676) through the Department of Molecular Physiology and Biophysics at Baylor College of Medicine. We thank Guanghui Liu, MD, PhD, Jun Song, MD, PhD, and Mary T. Nguyen for assisting with immunostaining. We thank Ludivine Russell and Laura C. Palmero for assistance in patient enrollment and tissue collection. We gratefully acknowledge Scott A. Weldon, MA, CMI, of Baylor College of Medicine, for assistance with illustrations, and Stephen N. Palmer, PhD, Rebecca Bartow, PhD, and Heather Leibrecht, MS, of the Texas Heart Institute, for providing editorial support.

\section{Comment on this Article or Ask a Question}

\section{References}

1. Centers for Disease Control and Prevention. National Center for Health Statistics. Compressed Mortality File 1999-2007. CDC WONDER On-line Database, compiled from Compressed Mortality File 1999-2007 Series 20 No. 2M, 2010. 2007.

2. Schlatmann TJ, Becker AE. Pathogenesis of dissecting aneurysm of aorta. Comparative histopathologic study of significance of medial changes. Am J Cardiol. 1977;39:21-26. 10.1016/S0002-9149(77)80005-2

3. Schlatmann TJ, Becker AE. Histologic changes in the normal aging aorta: implications for dissecting aortic aneurysm. Am J Cardiol. 1977:39:13-20. 10.1016/S00029149(77)80004-0

4. Mitchell RN, Libby P. Vascular remodeling in transplant vasculopathy. Circ Res. 2007;100:967978. 10.1161/01.RES.0000261982.76892.09

5. He R, Guo DC, Estrera AL, Safi HJ, Huynh TT, Yin $Z$, et al. Characterization of the inflammatory and apoptotic cells in the aortas of patients with ascending thoracic aortic aneurysms and dissections. J Thorac Cardiovasc Surg. 2006;131:671-678.e2. 10.1016/j. jtcvs.2005.09.018

6. He R, Guo DC, Sun W, Papke CL, Duraisamy $\mathrm{S}$, Estrera, AL, et al. Characterization of the inflammatory cells in ascending thoracic aortic aneurysms in patients with Marfan syndrome, familial thoracic aortic aneurysms, and sporadic aneurysms. J Thorac Cardiovasc Surg. 2008;136:922-929e1. 10.1016/j. jtcvs.2007.12.063

7. Thompson RW, Holmes DR, Mertens RA, Liao S, Botney MD, Mecham RP, et al. Production and localization of 92-kilodalton gelatinase in abdominal aortic aneurysms. An elastolytic metalloproteinase expressed by aneurysm-infiltrating macrophages. J Clin Invest. 1995;96:318-326. 10.1172/JCI118037

8. Eliason JL, Hannawa KK, Ailawadi G, Sinha I, Ford JW, Deogracias MP, et al. Neutrophil depletion inhibits experimental abdominal aortic aneu- rysm formation. Circulation. 2005;112:232-240. 10.1161/CIRCULATIONAHA.104.517391

9. Xiong W, Zhao Y, Prall A, Greiner TC, Baxter BT. Key roles of CD4+ T cells and IFNgamma in the development of abdominal aortic aneurysms in a murine model. J Immunol. 2004;172:2607-2612.

10. Majesky MW, Dong XR, Hoglund V, Mahoney WM, Daum G. The adventitia: a dynamic interface containing resident progenitor cells. Arterioscler Thromb Vasc Biol. 2011;31:1530-1539. 10.1161/ATVBAHA.110. 221549

11. Tieu $B C$, Lee $C$, Sun $H$, LeJeune $W$, Recinos A, Ju X, et al. An adventitial IL-6/MCP1 amplification loop accelerates macrophage-mediated vascular inflammation leading to aortic dissection in mice. J Clin Invest. 2009;119:3637-3651. 10.1172/ $\mathrm{JCl} 38308$

12. Andreeva ER, Pugach IM, Orekhov AN. Subendothelial smooth muscle cells of human 
aorta express macrophage antigen in situ and in vitro. Atherosclerosis. 1997;135:1927. 10.1016/S0021-9150(97)00136-6

13. Duffield JS. The inflammatory macrophage: a story of Jekyll and Hyde. Clin Sci. 2003;104: 27-38. 10.1042/CS20020240

14. Phillipson M, Kubes $P$. The neutrophil in vascular inflammation. Nat Med. 2011;17:13811390. 10.1038/nm.2514

15. Abdul-Hussien $\mathrm{H}$, Hanemaaijer R, Verheijen $\mathrm{JH}$ van Bockel JH, Geelkerken RH, Lindeman JH. Doxycycline therapy for abdominal aneurysm: Improved proteolytic balance through reduced neutrophil content. J Vasc Surg. 2009; 49:741-749. 10.1016/j.jvs.2008.09.055

16. Cohen JR, Parikh S, Grella L, Sarfati I, Corbie $\mathrm{G}$, Danna D, et al. Role of the neutrophil in abdominal aortic aneurysm development. Cardiovasc Surg. 1993;1:373-376.

17. Li M, Liu K, Michalicek J, Angus JA, Hunt JE, Dell'Italia $L$, et al. Involvement of chymasemediated angiotensin II generation in blood pressure regulation. J Clin Invest. 2004;114: 112-120. 10.1172/JCI200420805
18. Tchougounova E, Lundequist A, Fajardo I, Winberg JO, Abrink M, Pejler G. A key role for mast cell chymase in the activation of promatrix metalloprotease-9 and pro-matrix metalloprotease-2. J Biol Chem. 2005;280: 9291-9296. 10.1074/jbc.M410396200

19. Zhang J, Sun J, Lindholt JS, Sukhova GK, Sinnamon $M$, Stevens $R L$, et al. Mast cell tryptase deficiency attenuates mouse abdominal aortic aneurysm formation. Circ Res. 2011;108:1316-1327. 10.1161/CIRCRESAHA. 111.243758

20. Tsuruda T, Kato J, Hatakeyama K, Kojima K, Yano M, Yano Y, et al. Adventitial mast cells contribute to pathogenesis in the progression of abdominal aortic aneurysm. Circ Res. 2008;102:1368-1377. 10.1161/CIRCRESAHA. 108.173682

21. Baram D, Vaday GG, Salamon P, Drucker I, Hershkoviz R, Mekori YA. Human mast cells release metalloproteinase-9 on contact with activated $\mathrm{T}$ cells: juxtacrine regulation by TNF-alpha. J Immunol. 2001;167:4008-4016. 22. Maiellaro K, Taylor WR. The role of the adventitia in vascular inflammation. Cardiovasc
Res. 2007;75:640-648. 10.1016/j.cardiores. 2007.06.023

23. Zhao L, Moos MP, Gräbner R, Pédrono F, Fan J, Kaiser B, et al. The 5-lipoxygenase pathway promotes pathogenesis of hyperlipidemiadependent aortic aneurysm. Nat Med. 2004; 10:966-973. 10.1038/nm1099

24. Hiraguchi Y, Nagao M, Hosoki K, Tokuda R, Fujisawa T. Neutrophil proteases activate eosinophil function in vitro. Int Arch Allergy Immunol. 2008;146 Suppl 1:16-21. 10.1159/ 000126055

25. Xu L, Burke A. Acute medial dissection of the ascending aorta: evolution of reactive histologic changes. Am J Surg Pathol. 2013;37: 1275-1282. 10.1097/PAS.0b013e318294adc3

Cite this article as: $\mathrm{Wu} D$, Choi JC, Sameri A, Minard CG, Coselli JS, Shen YH, LeMaire SA. Inflammatory Cell Infiltrates in Acute and Chronic Thoracic Aortic Dissection. Aorta 2013;1(6):259-267. DOI: http://dx.doi. org/10.12945/j.aorta.2013.13-044

\section{EDITOR'S COMMENTS AND QUESTIONS}

\section{Editor's Comments:}

The authors are to be congratulated on this important study, making stronger the link between inflammation, aortic aneurysm, and aortic dissection.

\section{Editor's Questions:}

1. Why did you sample only the outer layer in your dissection patients? We know the dissection occurs in mid-media. Why not sample and examine the inner layer as well?

We are primarily interested in what drives aortic dilatation after aortic dissection has occurred. We focused on the outer wall of the false lumen because this is the region primarily responsible for aneurysm expansion and rupture in patients with dissection, and the region that would be the target for pharmacologic treatment designed to prevent dilatation after dissection. Changes involving the inner dissecting membrane (or dissection "flap") would have limited clinical relevance in chronic dissection.

2. Is it fair to include a preponderance of descending dissections as your control group when the acute dissections were all ascending? Information is mounting that ascending and descending dissections are different diseases (embryology, genetics, morphology, pathophysiology $[1,2]$, so one worries to have a disparate control group.

The editor makes a valid point. Our goal was to see whether the inflammatory response persists in chronic dissection patients. A set of patients with acute descending thoracic aortic dissection would be the ideal and proper controls for the group of patients with chronic descending thoracic aortic dissection; however, because it is very rare that these patients require open operative intervention, acutely dissected descending thoracic aortic tissue was not available for analysis. Although we acknowledge that acutely dissected aorta is not the ideal control, we believe it is a reasonable alternative at this stage, given that the inflammatory response to the tissue injury caused by acute dissection may be similar in different segments of the aorta, despite differences in underlying embryology, morphology, and pathophysiology; this supposition will require investigation.

3. You indicate that your controls were organ donors. Why did they have so much diabetes and stroke?

This was not intentional. Our main inclusion criterion for controls was that they had no evidence of aortic disease. Many of the control subjects were donors of non-vital organs and tissues. Selecting a control group with an age that matched the age of 
our patient population (>65 yr) resulted in a relatively high prevalence of comorbidities.

4. Is it fair to say that your hypothesis that chronic dissection patients would have less inflammation than acute dissection patients was not borne out? Any comments on this?

Our findings did not support our original hypothesis. In inflammation, a basic tenet is that after 24-48 hours, the predominant inflammatory cell infiltrate is macrophages, which progressively phases out over the next 7-14 days. We were surprised that the entire spectrum of inflammatory cells was present even in chronic dissection tissue samples, suggesting a sustained active inflammatory response.

5. Does the association you show us between inflammation and dissection inform us about causation? That is to say, which is the chicken and which is the egg? Does the inflammation come first, or the dissection?
We do not believe our findings provide information about the role of inflammation in the initiation of aortic dissection. Although aortic wall inflammation may certainly be a factor in the initial intimal/medial tear from which the dissection propagates, we purposely focused on tissue from the outer wall of the false lumen distal to the initial entry site to better understand the inflammatory response to dissection. We view the acute longitudinal splitting of the media as a form of severe vascular wall trauma that would be expected to spark a major acute inflammatory response, and we were particularly interested in how the inflammatory cell profile might change when moving into the chronic phase. Our findings suggest that a continued inflammatory response may contribute to progressive weakening of the outer aortic wall in patients who develop aneurysms caused by chronic dissection.

\section{References}

1. Elefteriades JA, Farkas EA. Thoracic aortic aneurysm clinically pertinent controversies and uncertainties. J Am Coll Cardiol. 2010; 55: 841-857. 10.1016/j.jacc.2009.08.084
2. Ruddy JM, Jones JA, Ikonomidis JS. Pathophysiology of thoracic aortic aneurysm (TAA): is it not one uniform aorta? Role of embryo- logic origin. Prog Cardiovasc Dis. 2013; 56: 68-73. 10.1016/j.pcad.2013.04.002 